\title{
Effects of silencing Rab27a gene on biological characteristics and chemosensitivity of non-small cell lung cancer
}

\author{
Xia Li ${ }^{4, *}$, Haiying Wang ${ }^{1, *}$, Qinggan $\mathrm{Ni}^{5, *}$, Zhiyuan Tang ${ }^{1}$, Jun $\mathrm{Ni}^{2}$, Liqin $\mathrm{Xu}^{1}$, Hua \\ Huang ${ }^{3}$, Songshi $\mathbf{N i}^{1}$ and Jian Feng ${ }^{1}$ \\ ${ }^{1}$ Department of Respiratory, Affiliated Hospital of Nantong University, Nantong 226001, Jiangsu, China \\ ${ }^{2}$ Department of Rehabilitation, Affiliated Hospital of Nantong University, Nantong 226001, Jiangsu, China \\ ${ }^{3}$ Department of Pathology, Affiliated Hospital of Nantong University, Nantong 226001, Jiangsu, China \\ ${ }^{4}$ Department of Respiratory, Yancheng Third People's Hospital, Yancheng 224002, Jiangsu, China \\ ${ }^{5}$ Department of Central Laboratory, Affiliated Hospital of Nantong University, Nantong 226001, Jiangsu, China \\ *These authors have contributed equally to this work \\ Correspondence to: Jian Feng, email: doctorfeng50@126.com \\ Keywords: non-small cell lung cancer, Rab27a, biological characteristics, proliferation, migration \\ Received: July 25, $2017 \quad$ Accepted: September 08, 2017 Published: October 10, 2017 \\ Copyright: Li et al. This is an open-access article distributed under the terms of the Creative Commons Attribution License 3.0 (CC \\ BY 3.0), which permits unrestricted use, distribution, and reproduction in any medium, provided the original author and source are \\ credited.
}

\section{ABSTRACT}

Rab27a, a member of the Rab protein family, can regulate the tumor microenvironment and promote the development of the tumor. Elevated expression of Rab27a is closely connected with many human cancers containing non-small cell lung cancer (NSCLC). But the role of Rab27a in non-small cell lung cancer and its possible mechanism is particularly unclear. In this research, we explored the effect of silencing Rab27a in vitro and in vivo, furnishing evidence that Rab27a could be a potential therapeutic target in NSCLC. Compared with corresponding control cells, silencing Rab27a had decreased ability of cell proliferation, migration and invasion in vitro and slower growth of xenograft tumors in mice. The expressions of apoptosis-associated proteins were induced with a reduction of anti-apoptotic protein in the NSCLC cells down-regulated Rab27a. Furthermore, Rab27a was associated with resistance to conventional chemotherapeutic agents. Our findings suggested that Rab27a might play a critical role in increasing chemosensitivity in NSCLC.

\section{INTRODUCTION}

Lung cancer is the most common respiratory tract malignancy with the top incidence and mortality. A variety of factors contribute to the occurrence and development of lung cancer. The death rate of lung cancer in developed countries continues to hold the top spot, while in developing countries that is also increasing year by year. Depending on the histology, stage of lung cancer, and general condition of the patients, the principles of multi-modality treatment including surgery, irradiation, chemotherapy, as well as bio-therapy and gene therapy are extensively discussed [1-3]. Even so, cancer metastasis and drug resistance after systematic treatment is still a major problem for patients with lung cancer [4]. Prognosis of patients with lung cancer is ominous, with a 5-year survival rate less than $15 \%$ [5].Therefore, recent researchs of lung cancer are mainly concentrated on the identification of biomarkers in patients and the development of molecularly targeted drugs [6].

shRNA (short hairpin RNA) is a expression vector in which double-stranded RNA degrades its homological mRNA and leads to sequence specific post-transcriptional gene silencing in an organism [7]. With the development of technology, silencing targeted genes associated with the progress of lung cancer is a new hope for patients with lung cancer [8]. 
The Rab family consists of more than 60 members and is widely expressed in mammals. The Rab27a family, molecular weight from 20 to $30 \mathrm{kDa}$, plays a significant role in endocytosis, cell secretion, growth and signal transduction. Rab27a, a member of the Rab family, can regulate the tumor microenvironment and promote tumor growth. Recently, some studies have found that Rab27a has a close relationship with many human cancers, Rab27a overexpression prefigures unfavourable prognosis in colorectal cancer [9-12]. Rab27a promotes the invasion and metastasis of human breast cancer cells [13]. However, the association between Rab27a and NSCLC is not very clear. We conducted the present research to thoroughly explore the function of Rab27a in NSCLC development.

Here in this article, in order to illustrate the relationship between Rab27a and NSCLC, we silenced Rab27a gene of NSCLC cells, further explored the changes in cell invasion, migration, proliferation and apoptosis in vitro and tumor model of human NSCLC cells in nude mice. Our intent is to search for potential targets related to NSCLC and thus to provide evidence for early diagnosis and treatment of NSCLC.

\section{RESULTS}

\section{The expression of Rab27a associated in NSCLC}

Our previous study showed Rab27a overexpression in the tissue of NSCLC. Here we tested Rab27a expression in four NSCLC lines (SPC-A-1, A549, H1650, H1975). Western blot results revealed that SPC-A-1 and H1650 cell lines expressed higher levels of Rab27a than H1975 and A549 cell lines (Figure 1A and 1B).

\section{The effect of silencing the expression of Rab27a on cell proliferation, migration and metastasis of NSCLC}

Next we showed the functional of transfecting the Rab27a expressions in NSCLC cell lines by testing three different sequences of siRNA targeting Rab27a, negative control and without any intervention control siRNA. Western blot proved Rab27a\#1 was the most convincing silent sequence (Figure 2A). We transfected the shRNA into H1650 and SPC-A-1 cell lines, for these two kinds of cell lines showed higher expression of Rab27a.

We next evaluated the ability of invasion, migration and proliferation in SPC-A-1 and H1650 cell lines. We found that after silenced with Rab27a shRNA (SPC-A-1-Rab27a and H1650-Rab27a) the proliferative capacity of cell lines was lower than the control groups (Figure 2B). In the experiment of invasion and migration, compared with the two control groups, fewer SPC-A-1-Rab27a and H1650-Rab27a cells migrated via the membrane in the migration chamber with or without the Transwell-precoated Matrigel, with statistical significance (Figure 2, C-E $\mathrm{P}<0.05$ ). The results of these experiments, silencing Rab27a expression can be effectively reduced the ability of proliferation, migration and invasion in NSCLC cells.

\section{Component activation and silencing of Rab27a on growth of NSCLC in nude mice}

The effect of Rab27a on tumor growth was studied by injecting different cell lines into BALB/c athymic nude mice subcutaneous to construct xenografts model. We first examined tumor growth in negative control and normal control groups. There was no significant difference in tumor growth curve at each point in time (Figure 3A and 3B $P>0.05, \mathrm{~A}: P=0.436, \mathrm{~B}: P=0.728)$. Thereafter, cells in which the high expression of Rab27a was silenced (H1650-Rab27a and SPC-A-1-Rab27a), their accordant control cells (H1650-scr and SPC-A-1-scr) and normal control cells were injected into the sides of the lower edge of the ribs subcutaneous, and tumor size was measured and registered every three days. Tumor growth curves demonstrated that the H1650-scr and SPC-A-1-scr cells of tumor volume after silenced for Rab27a expression was much lower than corresponding control and normal control. The average tumor volume every mouse after injection of H1650-Rab27a cells was approximately 720 $\mathrm{mm}^{3}$ at 31 days. The average tumor volume every mouse after injection of H1650-scr cells was approximately was $2512 \pm 172 \mathrm{~mm}^{3}$ at 31 days. There was significant difference between the two groups $(\mathrm{P}<0.05)$. Compared with the average tumor volume by SPC-A-1-scr (1985 $\left.\pm 182 \mathrm{~mm}^{3}\right)$, the average tumor volume by SPC-A$1-\mathrm{Rab} 27 \mathrm{a}$ was reduced $\left(562 \pm 120 \mathrm{~mm}^{3}\right) \quad(\mathrm{P}<0.05)$. Particularly, the tumor size of the mice that received stable transfection cell and cisplatin were significantly smaller than those only received one alone $(P<0.05)$. Interestingly, we found an interesting phenomenon that tumor growth speed was significantly expedited at certain periods (22 d in H1650 and $25 \mathrm{~d}$ in SPC-A-1).

\section{Expression and apoptosis of the Rab27a in vitro and vivo experiments}

By studying the expression of apoptosis protein after Rab27a silencing, the intrinsic mechanism of Rab27a induced growth of lung cancer cells was derived. Apoptotic protein caspase -3, caspe-9 and Bax expression were higher in spc-a-1-rab27a and h1650-rab27a cells than the corresponding control group and the normal control group. By comparison, the expression of anti-apoptotic protein Bcl-2 was reduced after Rab27a silencing,(Figure $4 \mathrm{~A}$ and $4 \mathrm{~B}$ ). Those results indicated that silencing Rab27a expression could enhance apotosis in NSCLC cells, and that should be explored in future researches.

We injected cisplatin into three of the eight mice from Rab27a silenced groups by intraperitoneal injection. 
We found that the level of tumor tissue apoptosis was higher than the normal control group after cisplatin treatment, in addition, the combination of silencing and cisplatin treatment, the tumor tissue apoptosis level was significantly increased (Figure 5A). For immunohistochemistry staining, Bax, Caspase-3, Fas-L,
Bcl-2 were tested on TMA. Observation of different levels of protein staining in the nucleus. The expression of Bax, caspase -3 and fasl was increased in h1650 - rab27a and h1650 - rab27a groups, and the expression of bcl - 2 was decreased. The number of apoptotic cells was the highest in shRNA and cisplatin combined tissues (Figure 5B).

A

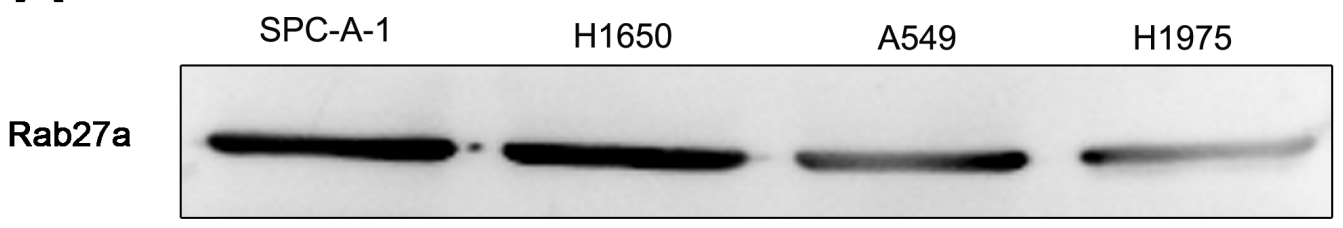

$28 \mathrm{KD}$

$\beta$-actin

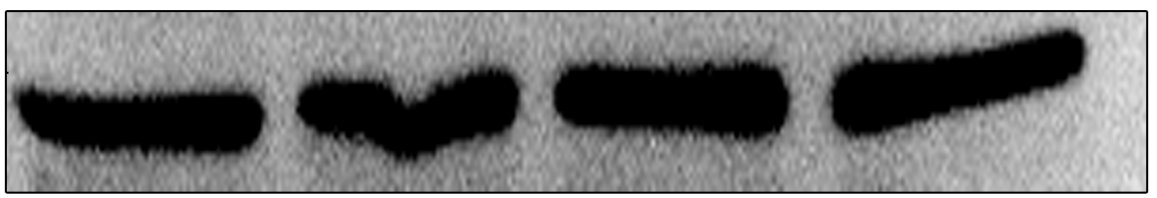

42KD

B

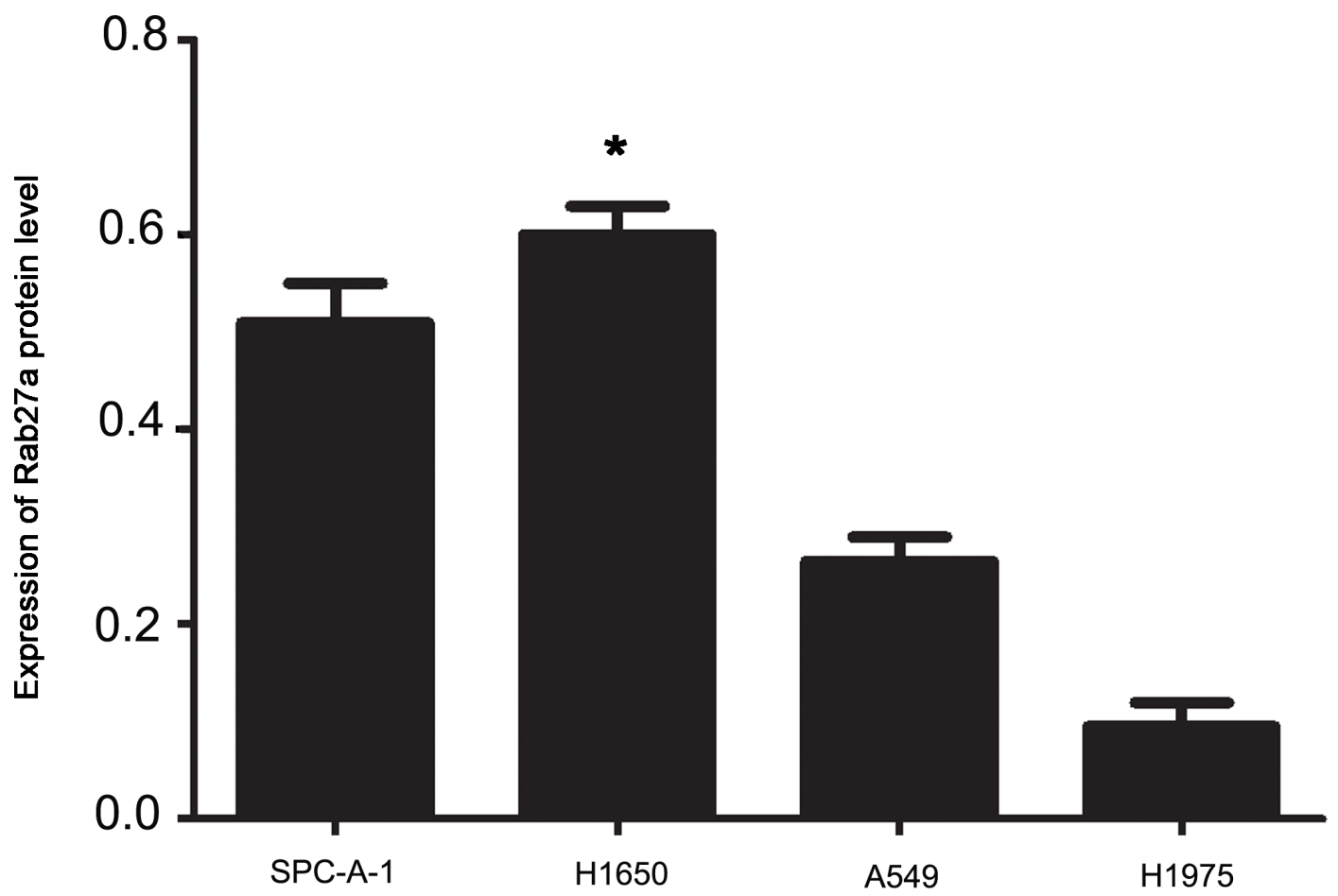

Figure 1: Expression of Rab27a protein in four non-small cell lung cancer cell lines. (A and $B$ ) $\beta$-actin was used as loading control. 

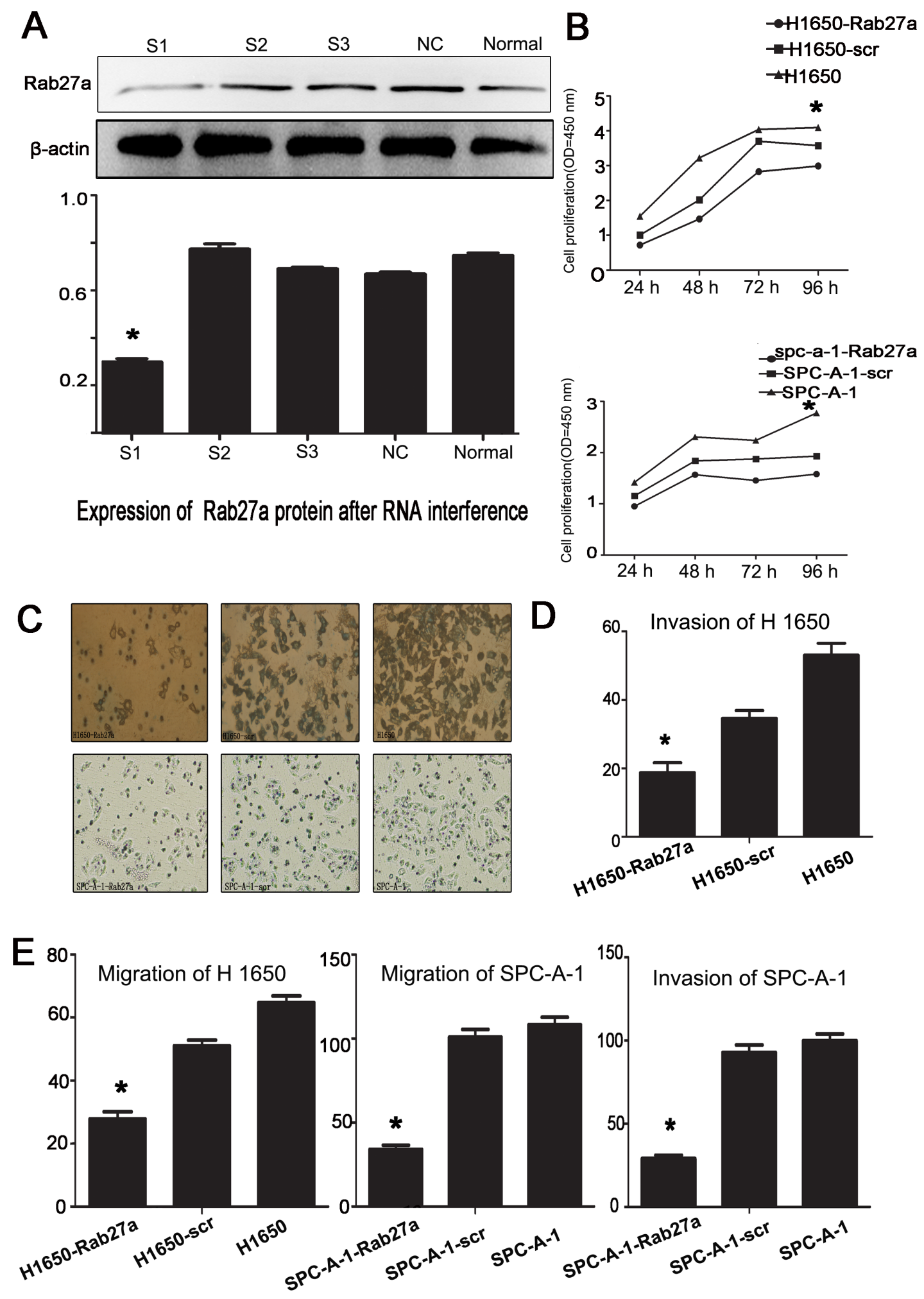

Figure 2: Effect of depleting or enforcing the expression of Rab27a on cell proliferation, migration and invasiveness of lung carcinoma cells. (A) western blot were used to select the most effective silencing shRNA targeting human Rab27a. (B) The proliferation ability of the four experimental cell lines was examined using CCK- 8 at $450 \mathrm{~nm}$. Specifically, $5 \times 10^{3}$ cells were seeded in 100 $\mu \mathrm{L}$ of medium per well into 96-well plates (three wells per each group). Then, $10 \mu \mathrm{L}$ of CCK8 solution was added to the culture medium in each well after $24 \mathrm{~h}, 48 \mathrm{~h}, 72 \mathrm{~h}$ and $96 \mathrm{~h}$. Then cells were incubated for another $3 \mathrm{~h}$. The absorbance was determined at $450 \mathrm{~nm}$ wavelength. (C-E) Migration and invasion ability were presented as total number of cells that migrated to the bottom chamber without or with the transwell-precoated matrigel, respectively, as calculated in at least six random fields (total magnification $\times 200)$ per filter. $\left({ }^{*}, \mathrm{P}<0.05\right.$ ). 
These results suggest that silencing Rab27a may positively regulate the apoptosis of NSCLC, whereas the use of cisplatin and targeting Rab27a binds more efficiently to promote apoptosis. We will continue to explore their mechanisms in future studies.

\section{Rab27a expression confers promoting chemotherapy-induced apoptosis}

24 hours after transfection, different concentrations of cisplatin were added in SPC-A-1-Rab27a and H1650Rab27a cells, corresponding and normal controls cells,

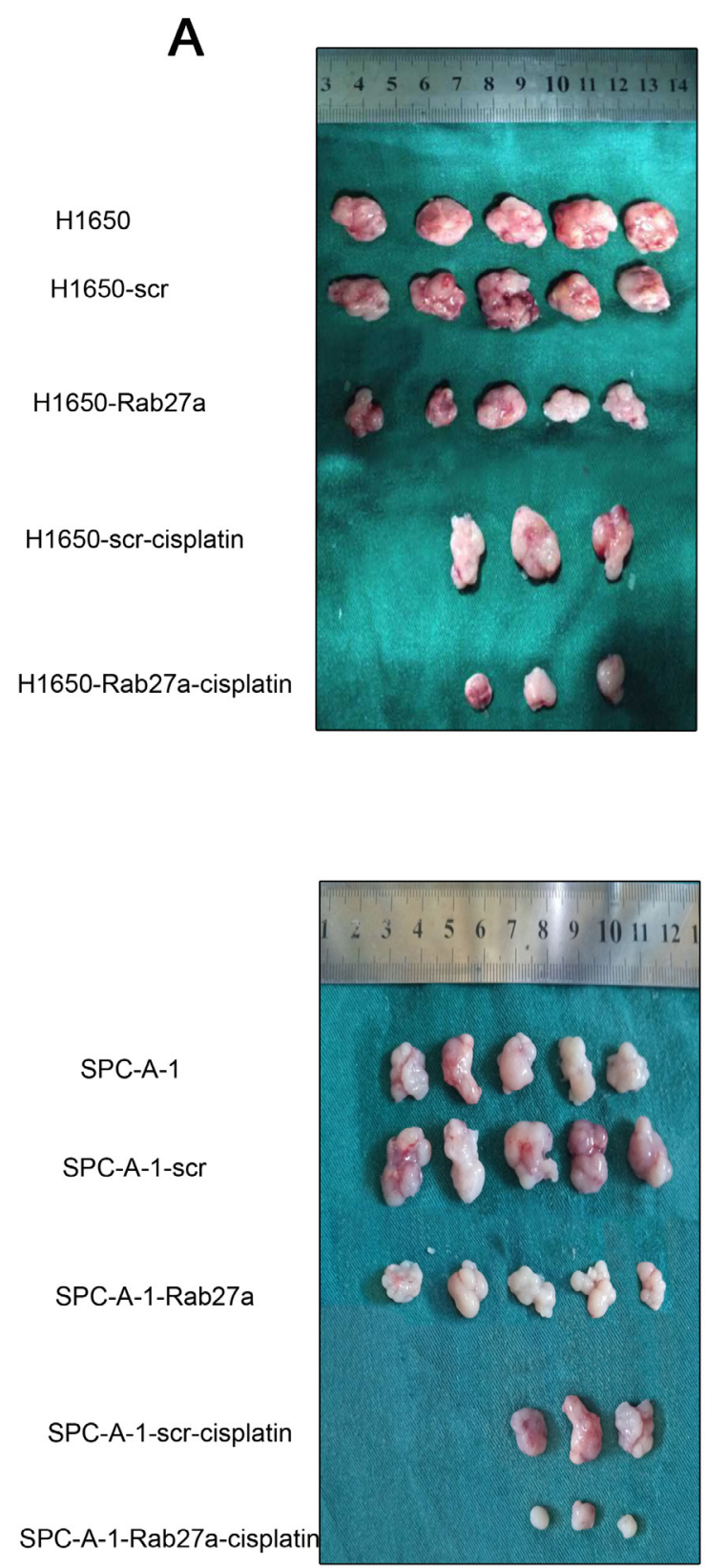

B
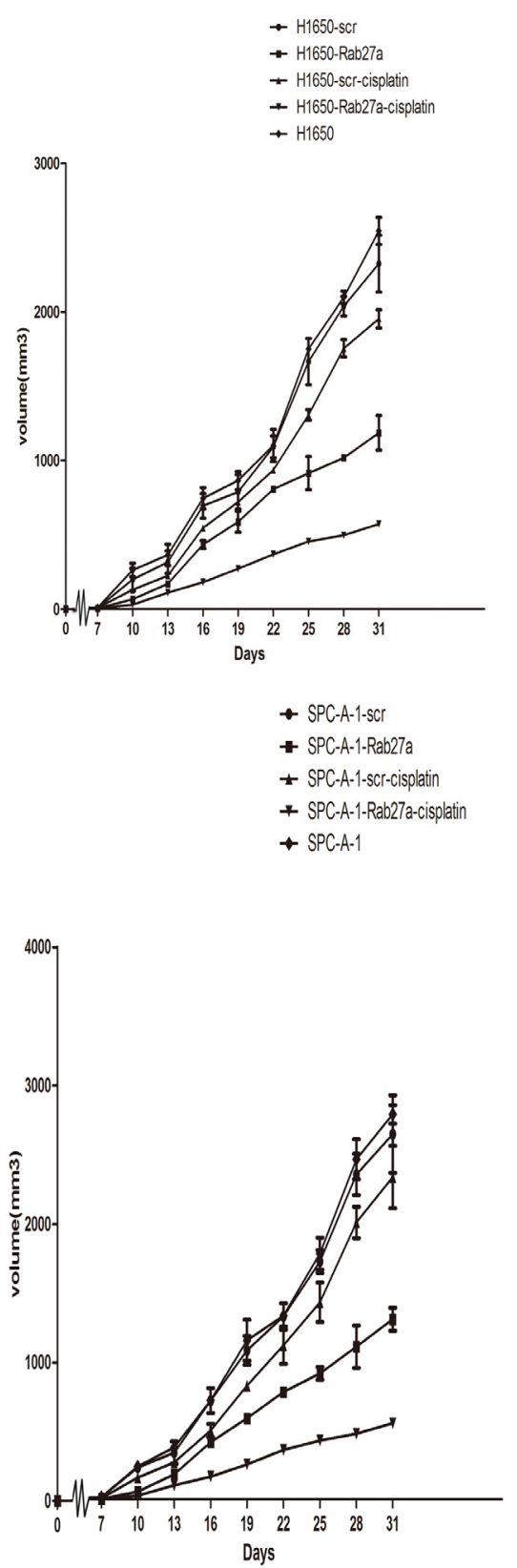

Figure 3: Effects of constitutive activation or silencing of Rab27a on growth of NSCLC in nude mice. Experimental cells were subcutaneously injected into BALB/c nude mice. (A) Images of tumors in normal control group, Rab27a silenced groups, corresponding controls groups and including intraperitoneal injection of cisplatin groups. (B) The tumor size were measured every third day after inoculation, growth of tumors produced by subcutaneous injection of mice with H1650, H1650-scr, H1650-Rab27a, then H1650-scr, H1650-Rab27a treated by cisplatin. The tumors from mice that received cisplatin and stable transfection cell injection were smaller than those either treated with cisplatin or transfected alone. All date are shown as mean $\pm \mathrm{SD}$. ${ }^{*}, P<0.05$. 
then we observed the three group cells by using the method named CCK8 after 48 hours and detected the changes of sensitivity in SPC-A-1 and H1650 cells to cisplatin. The results showed that the sensitivity of SPC-A-1-Rab27a and H1650-Rab27a cells to chemotherapeutic drugs increased significantly compared with the corresponding controls and normal controls $(\mathrm{P}<0.05)$. It showed that the target Rab27a gene could increase drug sensitivity of lung

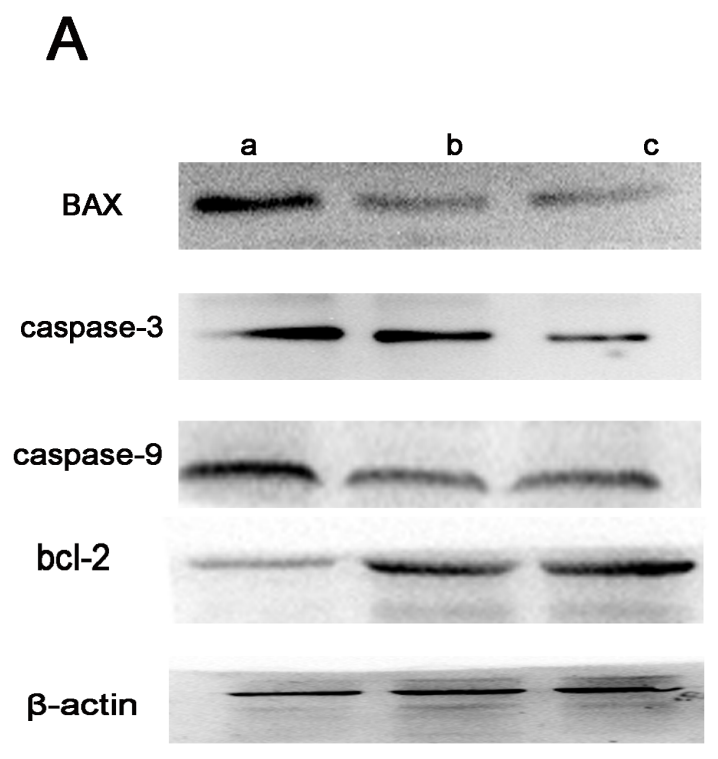

a:H1650-Rab27a

b:H1650-scr

c:H1650

B

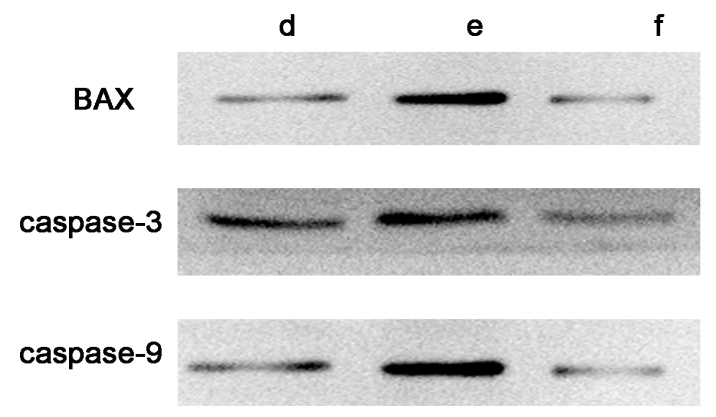

bcl-2

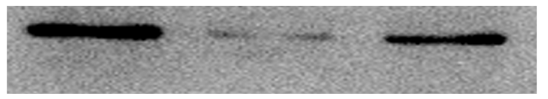

$\beta$-actin

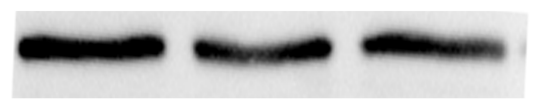

d:SPC-A-1-scr

e:SPC-A-1-Rab27a

f:SPC-A-1
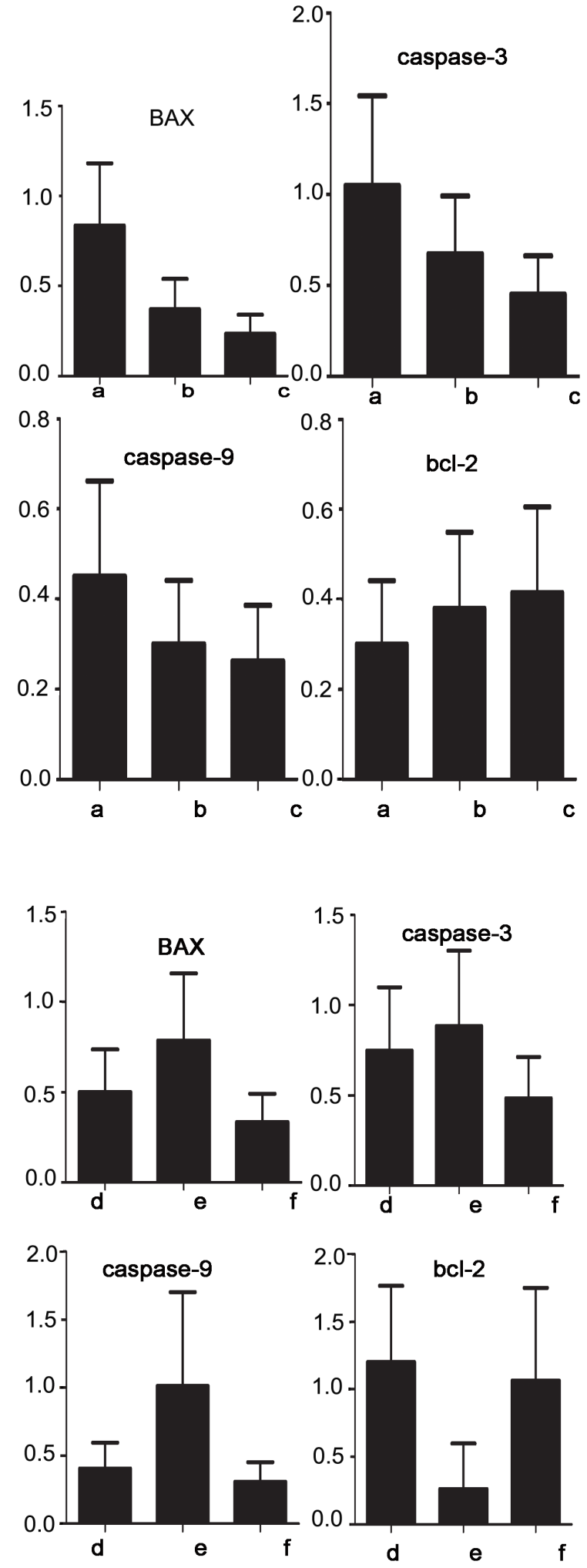

Figure 4: Rab27a expression and apoptosis in vitro. (A and B) Western blot analysis of apoptosis-associated protein expression in Rab27a silenced cells and tissues. $\beta$-actin was used as a control. 
cancer cells (Figure 6). We investigated the relationship between Rab27a expression and chemoresistance to four chemotherapeutic agents in NSCLC cells and examined whether Rab27a could regulate drug-induced apoptosis. The depletion of Rab27a in h1650 - Rab27a and SPCA-1-Rab27a cells leads to an increased response to chemotherapy in NSCLC (Figure 6, A: $\mathrm{P}=0.004$ and $\mathrm{B}: \mathrm{P}=0.008)$. The IC50 in H1650-Rab27a and SPC-A$1-\mathrm{Rab} 27 \mathrm{a}$ cells ware lower than in control group cells

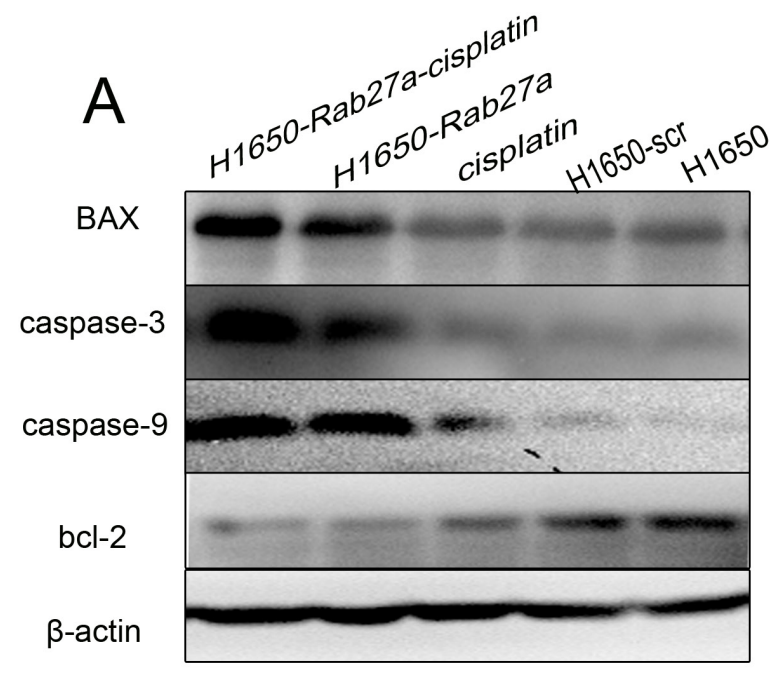

B
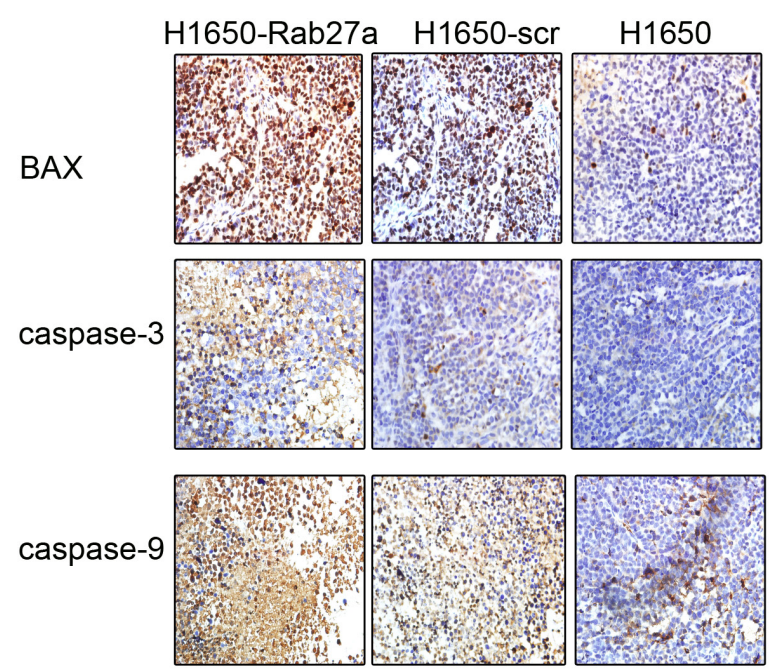

bcl-2
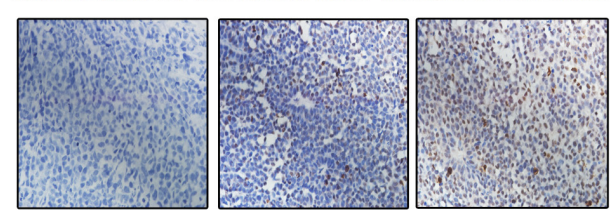

(Figure 6C and 6D $\mathrm{P}<0.05$ ). Thus, the downregulation of Rab27a can increase the sensitivity of NSCLC cells to chemotherapeutic agents, which may be related to above results.

\section{DISCUSSION}

The occurrence of tumor is the result of the interaction of multiple genes. The main reason for the

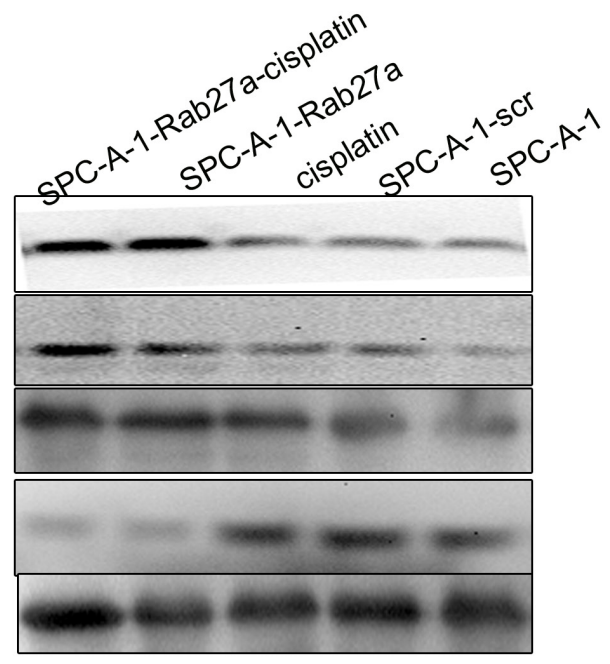

SPC-A-1-Rab27a SPC-A-1-scr SPC-A-1
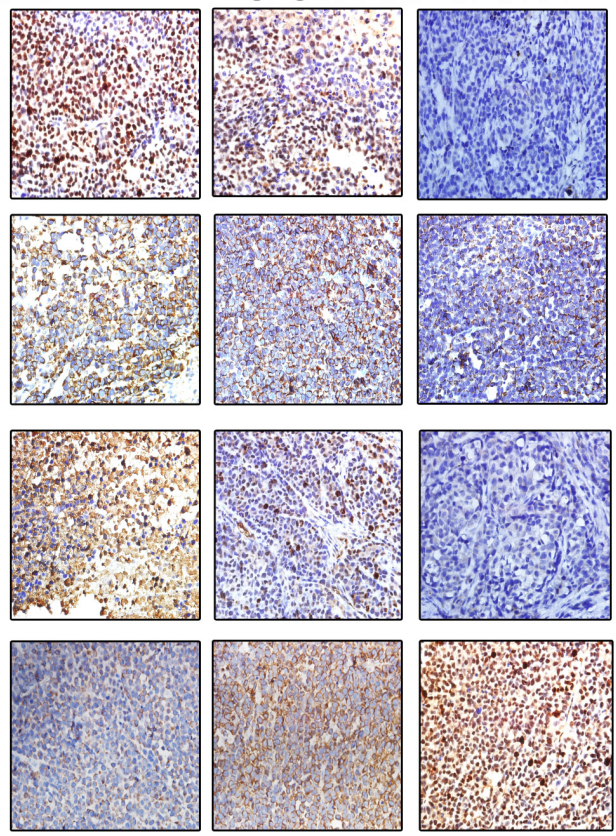

Figure 5: Rab27a expression and apoptosis in vitro. (A) Western blot of apoptosisassociated protein expression in frozen tissues, including upon cisplatin combination treatment. $\beta$-actin was used as a loading control. (B) Analysis of apoptosis-associated protein expression on TMA was performed by immunohistochemistry staining (magnification $\times 200$ ). The result was consistent with $(A)$. 
low survival rate of cancer patients is tumor metastasis and drug resistance. Tumor invasion of the surrounding tissue and the formation of distant metastases, resulting in other organ dysfunction and even organ failure, seriously affect the quality of life of patients and threaten the life of patients $[14,15]$. The molecular mechanism of tumor invasion and metastasis has long been the focus of tumor

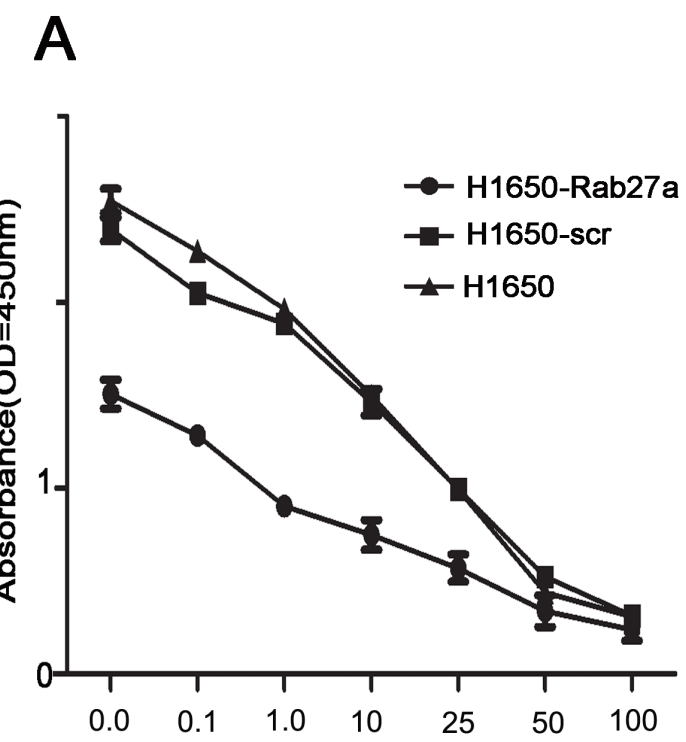

Cisplatin(nM)

B

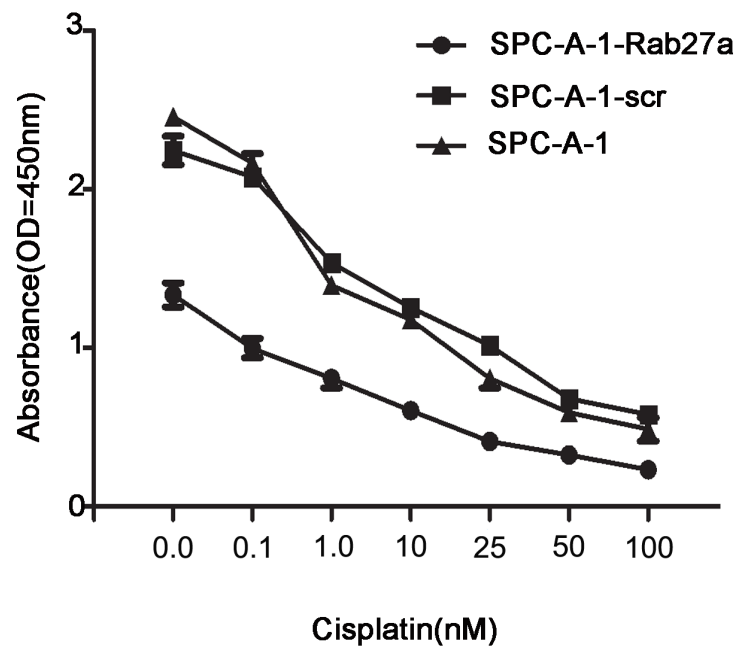

research. Lung cancer is very common all over the world at present. Because of the invasion and metastasis, the recurrence rate is very high even in patients undergoing radical operation for lung cancer. And $70 \%$ of the patients diagnosed with lung cancer have been advanced, although several traditional chemotherapy regimens may prolong the survival of patients, many patients can not tolerate

C

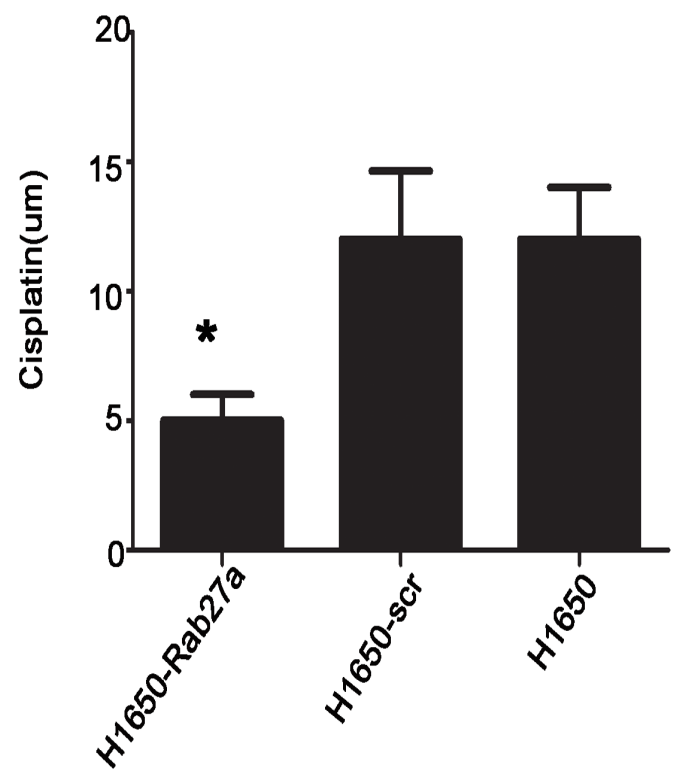

D

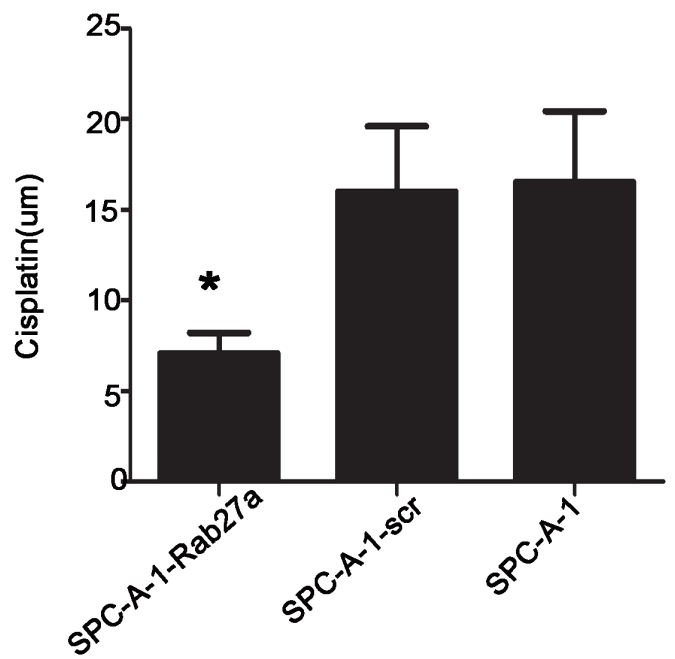

Figure 6: Rab27a expression increases sensitivity to chemotherapeutic drugs. (A-D) Rab27a depletion in SPCA-1-Rab27a and H1650-Rab27a cells contributed to increased apoptotic response to cisplatin. (Error bars $=95 \%$ confidence interval, $\mathrm{CI}$; $\mathrm{P}=0.003$ and $\mathrm{P}=0.007$ respectively). 
the side effects of chemotherapy [16-18]. So, finding new prognostic markers of lung cancer, exploring the mechanism of its partial mechanism, and taking targeted therapy are particularly important.

In recent years, significant progress has been made in the field of cancer genomics, especially in the occurrence and development of lung cancer, and the importance of epigenetic specificity has been widely recognized [19]. In the case that the nucleotide sequence of the gene is not changed, the epigenetic gene is a branch of genetics that expresses a genetic change. There are a lot of epigenetic phenomena, well-known as gene silencing, DNA methylation, nucleolar dominance, dormant transposon activation and genomic imprinting. Studying the changes of these epigenetic changes can identify some tumor related genes, and provide new insights and ideas for the treatment of lung cancer [20-22]. The topic chosen gene silencing technology, rapid and specific identification of target gene mRNA sequence, interference the protein synthesis, and down regulating the expression of target genes, obtaining target gene function loss or decreased expression, could achieve the purpose of disease treatment.

In this study, by RNA interference technology, we investigated the effects of Rab27a gene silencing on the biological characteristics of NSCLC and its role as a potential target for lung cancer treatment. At the cellular level, four human lung cancer cell lines were selected and screened by Western blot, and the high expression Rab27a cell lines were selected for subsequent silencing experiments. Western blot was applied to confirm the RNA interference technique that the gene silencing could affect the transcription and translation level thus reduce the expression of Rab27a, and the interference efficiency can reach $70 \%$. Then we used the cell proliferation assay (CCK8 method) to find that the proliferative ability of lung cancer cells was obviously decreased after gene silencing; the ability of invasion and migration of lung cancer cells, confirmed by transwell migration and invasion assay, were significantly decreased after Rab27a gene interference. These biological effects of Rab27a in NSCLC were in accordance with those reported in other cancers (breast cancer [23, 24], pancreatic cancer [25], colorectal cancer $[26,27]$ and so on). In addition, through Western blot, we found that Rab27a gene interference cells highly expressed apoptosis related Caspase-3, Bax, caspase- 9 expression compared with the control group, while the anti apoptotic protein bcl-2 expression was reduced, the differences were statistically significant $(\mathrm{P}<0.05)$. In a word, reduction of Rab27a expression significantly promotes the apoptosis of lung cancer cells, resulting in inhibition of tumor growth.

We also studied the changes of the sensitivity to chemotherapy in cells after Rab27a gene silencing. We found that tumor shown in animals on injection of Rb27a knockout do not appear to be significantly lower in size or weight. The tumors are still big, therefore $\mathrm{Rb} 27 \mathrm{a}$ may not be significant gene in regulation of tumor growth.
However, treatment of animals bearing tumors generated by $\mathrm{Rb} 27$ a knockout cells followed by cisplatin treatment show a significant reduction, therefore, Rb27a may be involved in changing the sensitivity of tumor cells for cisplatin, but whether directly involved in tumor growth is not clear. Therefore, the treatment focused on Rab27a gene could target multiple aspects of tumor treatment, for example, it could selectively increase the enzymatic activity of apoptosis gene and/or inhibit the expression of anti hole of apoptosis genes in tumor cells, strengthen the efficacy of chemotherapy or radiotherapy and reduce the toxic and side effects [28-30], thus provide a new reference for clinical chemotherapy drugs. So, it is extremely important to further explore the role of Rab27a in the regulation of NSCLC.

Our research results showed that in NSCLC cell level, interference gene Rab27a decreased significantly the proliferation and invasion of tumor, promoted apoptosis, and increased the sensitivity to chemotherapy drugs of NSCLC. Which provide experimental and theoretical basis for the treatment of lung cancer.

\section{MATERIALS AND METHODS}

\section{Cell lines and cell culture}

Human lung cancer cell lines A549, H1650, SPC-A-1 and H1975 were respectively purchased from the Shanghai Institute of Biochemistry and Cell biology, the Chinese Academy of Sciences. These four cell lines were cultured in medium (cyclone Chinese abstract, Logan City, Utah, USA) with 10\% fetal bovine serum (FBS), $2 \mathrm{~mm}$ L-glutamine, $100 \mathrm{U} / \mathrm{ml}$ Penicillin/Streptomycin mixture (GIBCO BRL, Grand Island, NY, USA) under a humid atmosphere in $5 \% \mathrm{CO}_{2}$ at $37^{\circ} \mathrm{C}$.

\section{Western blot}

Total protein of these tumour cells and tissues were extracted by a lysis buffer (Beyotime Institute of Biotechnology, Nantong, China), and protein concentration were measured by the BSA method (Beyotime Institute of Biotechnology, Nantong, China). An equal number of protein (40 $\mu \mathrm{g}$ per lane) were separated by SDSpolyacrylamide gel electrophoresis (PAGE) in 6\%, 10\% and $12 \%$ acrylamide gels and switched to poly vinblastine difluoride (PVDF) membranes (Millipore Corporation, USA) at $300 \mathrm{~mA}$ for two hours. The membrane was blocked in 5\% fat-free milk and incubated with the following primary antibodies overnight at $4^{\circ} \mathrm{C}$ : rabbit antiRab27a (1:3000 dilution; Abcam), rabbit anti-Bax (1:1000 dilution; Abcam), polygonal rabbit anti-Caspase3 (1:1000 dilution; Abcam), polygonal rabbit anti-Caspase9 (1:1000 dilution; Abcam) and monoclonal rabbit anti-Bcl-2 (1:500 dilution; Abcam). The secondary antibody was horseradish peroxidase-conjugated (HRP)-conjugated goat anti-rabbit 
antibody (1:1000, Beyotime Institute of Biotechnology). After stripping, the membrane was reproved with $\beta$-actin (1:1000, Beyotime Institute of Biotechnology) overnight at $4^{\circ} \mathrm{C}$, followed by incubation with secondary antibody as above at room temperature for $2 \mathrm{~h}$. Bands were visualised using an enhanced chemiluminescence system (ECL, Beyotime Institute of Biotechnology). Data were quantified by densitometry.

\section{shRNA transfection, plasmid constructs and generation of stable cell lines}

Three different shRNA sequences targeting human Rab27a and negative control shRNA were designed and obtained from Shanghai Genepharma Corporation. The sequences of Rab27 and control shRNA were as follows: Rab27a shRNA-1 sequence: 5'-CAGTGTACTTTACCAATA-3', site: 321-338, Rab27a shRNA-2 sequence: 5'-AGGAGAGGTTTCGTAGCT-3', site: 485-502, Rab27a shRNA-3 sequence: 5'-CTGCCAATGGGACAAACA-3', site: 743-760, negative control shRNA sequences: 5'-GGATCAGTTAAGTGAAGA-3', site: 873-890. Cells were then transfected by Lipofectamine ${ }^{\mathrm{TM}} 2000$ (Invitrogen) according to the manufacturer's instructions. The most effective silencing sequence was filtered by western blot, and then ligated into the PGPH1/GFP/ Neo vector. Full-length Rab27a cDNA was cloned into pCMV6/AC/GFP vector (OriGene, USA). Two cell lines were separately transfected with plasmids and selected by GFP sorting. Cells were then grown in complete medium containing $200 \mu \mathrm{g} / \mathrm{ml}$ G418 (Roche Diagnostics, Mannheim, Germany). Fluorescence microscope was utilized to confirm the transfection efficiency. Cells were separated and expanded into clones after four weeks. Subclass cells expressing Neo and Rab27a genes were named as H1650-Rab27a, SPC-A-1-Rab27a. The corresponding controls were named H1650-scr, SPC-A1 -scr.

\section{Cell proliferation assays}

For analysis of cell proliferation, SPC-A-1-Rab27a, H1650-Rab27a， SPC-A-1-scr, H1650-scr, SPC-A-1, H1650 cells and normal control cells $\left(5 \times 10^{3}\right)$ in 100 $\mu \mathrm{L}$ of medium were seeded per well into 96-well plates (three wells each group). Cell proliferation was evaluated using the Cell Counting Kit-8 (CCK-8, Beyotime Institute of Biotechnology) according to the manufacturer's instructions. Briefly, $10 \mu \mathrm{L}$ of CCK8 solution was added to the culture medium in each well, and cells were incubated for $3 \mathrm{~h}$. The absorbance was determined at 450 $\mathrm{nm}$ wavelength. The assays were repeated three times with triplicate samples.

\section{Transwell migration and invasion assays}

Ambers consisting of Transwell-precoated Matrigel with $8 \mu \mathrm{m}$ pores were inserted in 24-well tissue culture plates (BD Biosciences, Bedford, MA). Cells from different groups $\left(1 \times 10^{5}\right)$ were plated onto the top chamber in RMPI1640 without FBS and the bottom chamber was filled with RMPI1640 containing 10\% FBS as a chemoattractant. After $24 \mathrm{~h}$ of incubation in a $5 \% \mathrm{CO}_{2}$ humidified atmosphere at $37^{\circ} \mathrm{C}$, noninvading cells were removed by wiping the upper surface of the membrane with a cotton swab, and the filter membrane was fixed with $4 \%$ paraformaldehyde and stained with Exam MaSiLiang blue. The degree of invasion was quantified by counting the cells that had migrated through the membrane in at least six random fields (total magnification, $\times 200$ ) per filter. Experiments were repeated three times in triplicate. For analysis of cell migration, we use the modified Boyden Chambers without the Transwell-precoated Matrigel membrane filter, using the method performed as above.

\section{Tumor formation in nude mice}

H1650-Rab27a, SPC-A-1-Rab27a and corresponding control cells were injected subcutaneously in mice to investigate the ability to generate xenograft tumours. BALB/cathymic nude mice (4 to 6 weeks old) were purchased from Shanghai Laboratory Animal Center, China and kept in a specific pathogen-free environment. All mouse experiments followed institutional guidelines and were approved by the committee on the Ethics of Animal Experiments of Nantong University, Permit Number: SCXK (hu) 2012-0002. We harvested $1 \times 10^{7}$ cells by incubation in trypsin-EDTA, washed the cells twice with PBS, resuspended the cells in $0.2 \mathrm{~mL}$ of RMPI medium, and injected each cell line subcutaneously into $\mathrm{BALB} /$ cathymic nude mice. (Eight mice were used per cell line and each mouse received two injections, each of $1 \times 10^{7}$ cells, in the bilateral flank to form two tumors. We injected stably transfected cells into one side of each mouse and the corresponding control cells in the other side.) To explore the effect of altered Rab27a expression on chemotherapeutics, we selected the classical antitumor drug cisplatin. At 7 days of tumor formation, three of the eight mice in each group in which Rab27a was silenced were randomly selected and received an intraperitoneal injection of cisplatin $(7.5 \mathrm{mg} / \mathrm{kg})$. The remaining five mice received an injection of the same amount of saline. The tumor growth of different treatment groups was monitored until the day that mice were ended thier life. The date at which the first grossly visible tumor appeared was recorded, and the tumor size was measured every 3 days. Two-dimensional measurements were taken with an electronic caliper after injection, and the tumor volume was calculated with the following formula: tumor volume (in $\left.\mathrm{mm}^{3}\right)=\pi / 6 \times a \times \mathrm{b}^{2}$, where a was the longest diameter, 
and $\mathrm{b}$ was the shortest diameter. When a tumor reached $2.0 \mathrm{~cm}$ in diameter, the mouse was anesthetized by $1 \%$ pentobarbital sodium (Sigma, St. Louis, MO, USA) and photographed. The tumors were excised, weighed and measured. Half of the primary tumors were fixed in $10 \%$ formalin overnight and subjected to routine histological examination by investigators who were blinded to the tumor status. The other half was frozen at $-80^{\circ} \mathrm{C}$ for later research.

\section{Tumor tissue microarray (TMA) and immunohistochemistry (IHC) staining}

All tumor samples were embedded in paraffin after fixing in $10 \%$ formaldehyde for $24 \mathrm{~h}$ and used for constructing the TMA. A representative area of each sample was selected and $2.0 \mathrm{~mm}$ tissue cores were designed for constructing a TMA by Shanghai Super Biotek, China. We used hematoxylin-eosin staining (H\&E) to confirm the quality of TMA sections. IHC staining was performed as described previously. Briefly, sections (4 $\mu \mathrm{m})$ were deparaffinized and rehydrated. Antigen retrieval was performed by boiling under pressure in citrate buffer, $\mathrm{pH}$ 6.0, for $3 \mathrm{~min}$. Nonspecific binding was blocked by $5 \%$ goat serum in PBS for $15 \mathrm{~min}$, and the tissues were incubated with primary antibodies as follows: rabbit antiRab27a (1:300 dilution; Abcam), rabbit anti-Bax (1:300 dilution; Abcam), monoclonal rabbit anti-Bcl-2 (1:250 dilution; Abcam), polyclonal rabbit antiCaspase3 (1:300 dilution; Abcam) and monoclonal rabbit anti-Fas (1:250 dilution; Abcam). The secondary antibody was EnVision goat anti-rabbit HRP (DAKO, USA). The immunostained sections were evaluated by two trained pathologists who were unaware of our research purpose.

\section{Chemotherapeutic cell treatments}

Cisplatin (DDP) was used at $0.1-100 \mu \mathrm{M}$ to determine the half-maximal inhibitory concentration (IC50) values in cell lines in which Rab27a was silenced and their corresponding controls cells. Cells $\left(5 \times 10^{3}\right)$ were added to each well in a 96- well plate and cultured for $24 \mathrm{~h}$. Cells were then treated with drugs for $12 \mathrm{~h}$ and the media was replaced by fresh medium without drugs for additional $48 \mathrm{~h}$. Cell viability was measured by a Cell Counting Kit-8 (Beyotime Institute of Biotechnology) at $450 \mathrm{~nm}$ as above. DMSO treatment was used as a control. The survival of each cell line was compared with their corresponding control cell line. Assays were repeated three times.

\section{Statistical analysis}

All statistical analyses, including t-test, $\chi^{2}$-test, and Mann-Whitney U-test, were carried out with the GraphPad Prism software (version 5; GraphPad Software, La Jolla,
CA, USA) and STATA 9.0 software (Stata Corporation, College Station, TX).

\section{CONFLICTS OF INTEREST}

There are no conflicts of interest associated with this manuscript.

\section{FUNDING}

This study was supported by sponsored project of Jiangsu provincial Six Talent Peaks (2014-WSN-028) of Jiangsu Province, China.

\section{REFERENCES}

1. Tang H, Ge L, Shao W, Qiu Y, Cui D. Effect of targeted silencing of hTERT mRNA by lentivirus-mediated siRNA on A549 lung cancer cells in vitro. Mol Biol Rep. 2013; 40: 605-616.

2. Ellis PM, Vandermeer R. Delays in the diagnosis of lung cancer. J Thorac Dis. 2011; 3: 183-188.

3. Méndez M, Custodio A, Provencio M. New molecular targeted therapies for advanced non-small-cell lung cancer. J Thorac Dis. 2011; 3: 30-56.

4. Shih JY, Yang PC. The EMT regulator slug and lung carcinogenesis. Carcinogenesis. 2011; 32: 1299-1304.

5. Xiao Y, Li X, Wang H, Wen R, He J, Tang J. Epigenetic regulation of miR-129-2 and its effects on the proliferation and invasion in lung cancer cells. J Cell Mol Med. 2015; 19: 2172-2180.

6. Ramalingam SS, Owonikoko TK, Khuri FR. Lung cancer: new biological insights and recent therapeutic advances. CA Cancer J Clin. 2011; 61: 91-112.

7. Cho WY, Hong SH, Singh B, Islam MA, Lee S, Lee AY, Gankhuyag N, Kim JE, Yu KN, Kim KH, Park YC, Cho $\mathrm{CS}$, Cho MH. Suppression of tumour growth in lung cancer xenograft model mice by poly (sorbitol- co-PEI)- mediated delivery of osteopontin siRNA. Eur J Pharm Biopharm. 2015; 94: 450-462.

8. Zhang H, Li Z, Wang K, Ren P. Combined treatment of XIAP-targeting shRNA and celecoxib synergistically inhibits the tumour growth of non small cell lung cancer cells in vitro and in vivo. Oncol Rep. 2015; 33: 1079-1088.

9. Meeths M, Bryceson YT, Rudd E, Zheng C, Wood SM, Ramme K, Beutel K, Hasle H, Heilmann C, Hultenby K, Ljunggren HG, Fadeel B, Nordenskjöld M, Henter JI. Clinical presentation of Griscelli syndrome type 2 and spectrum of RAB27A mutations. Pediatr Blood Cancer. 2010; 54: 563-572.

10. Yamaoka M, Ishizaki T, Kimura T. Interplay between Rab27a effectors in pancreatic beta-cells. World J Diabetes. 2015; 6: 508-516. 
11. Shi C, Yang X, Ni Y, Hou N, Xu L, Zhan F, Zhu H, Xiong L, Chen P. High Rab27A expression indicates favourable prognosis in CRC. Diagn Pathol. 2015; 10: 68.

12. Bobrie A, Krumeich S, Reyal F, Recchi C, Moita LF, Seabra MC, Ostrowski M, Théry C. Rab27a supports exosomedependent and -independent mechanisms that modify the tumour microenvironment and can promote tumour progression. Cancer research. 2012; 72: 4920-4930.

13. Montel V, Huang TY, Mose E, Pestonjamasp K, Tarin D. Expression profiling of primary tumours and matched lymphatic and lung metastases in a xenogeneic breast cancer model. Am J Pathol. 2005; 166: 1565-1579.

14. Rooney C, Sethi T. The epithelial cell and lung cancer: the link between chronic obstructive pulmonary disease and lung cancer. Respiration. 2011; 81: 89-104.

15. Ulahannan SV, Brahmer JR. Antiangiogenic agents in combination with chemotherapy in patients with advanced non-small cell lung cancer. Cancer Invest. 2011; 29: 325-337.

16. Gao W, Liu L, Lu X, Shu Y. Circulating microRNAs: possible prediction biomarkers for personalized therapy of non-small-cell lung carcinoma. Clin Lung Cancer. 2011; 12: 14-17.

17. Smith CB, Kelley AS, Meier DE. Evidence for new standard of care in non-small cell lung cancer patients. Semin Thorac Cardiovasc Surg. 2010; 22: 193-194.

18. Yang P. Epidemiology of lung cancer prognosis: quantity and quality of life. Methods Mol Biol. 2009; 471: 469-486.

19. Piperi C, Vlastos F, Farmaki E, Martinet N, Papavassiliou AG. Epigenetic effects of lung cancer predisposing factors impact on clinical diagnosis and prognosis. J Cell Mol Med. 2008; 12: 1495-1501.

20. Tommasi S, Zheng A, Weninger A, Bates SE, Li XA, Wu X, Hollstein M, Besaratinia A. Mammalian cells acquire epigenetic hallmarks of human cancer during immortalization. Nucleic Acids Res. 2013; 41: 182-195.

21. Siddle HV, Kreiss A, Tovar C, Yuen CK, Cheng Y, Belov K, Swift K, Pearse AM, Hamede R, Jones ME, Skjødt K, Woods GM, Kaufman J. Reversible epigenetic downregulation of mhc molecules by devil facial tumour disease illustrates immune escape by a contagious cancer. Proc Natl Acad Sci U S A. 2013; 110: 5103-5108.
22. Cruickshanks HA, Vafadar-Isfahani N, Dunican DS, Lee A, Sproul D, Lund JN, Meehan RR, Tufarelli C. Expression of a large line-1-driven antisense rna is linked to epigenetic silencing of the metastasis suppressor gene tfpi-2 in cancer. Nucleic Acids Res. 2013; 41: 6857-6869.

23. Hendrix A, Maynard D, Pauwels P, Braems G, Denys H, Van den Broecke R, Lambert J, Van Belle S, Cocquyt V, Gespach C, Bracke M, Seabra MC, Gahl WA, et al. Effect of the secretory small gtpase rab27b on breast cancer growth, invasion, and metastasis. J Natl Cancer Inst. 2010; 102: 866-880.

24. Wang JS, Wang FB, Zhang QG, Shen ZZ, Shao ZM. Enhanced expression of rab27a gene by breast cancer cells promoting invasiveness and the metastasis potential by secretion of insulin-like growth factor-ii. Cell. 2010; 143: 1005-1017.

25. Wang Q, Ni Q, Wang X, Zhu H, Wang Z, Huang J. High expression of RAB27A and TP53 in pancreatic cancer predicts poor survival. Med Oncol. 2015; 32: 372.

26. Supiot S, Gouraud W, Campion L, Jezéquel P, Buecher B, Charrier J, Heymann MF, Mahé MA, Rio E, Chérel M. Early dynamic transcriptomic changes during preoperative radiotherapy in patients with rectal cancer: a feasibility study. World J Gastroenterol. 2013; 19: 3249-3254.

27. Dong W, Cui J, Yang J, Li W, Wang S, Wang X, Li X, Lu Y, Xiao W. Decreased expression of Rab27A and Rab27B correlates with metastasis and poor prognosis in colorectal cancer. Discov Med. 2015; 20: 357-367.

28. Voulgari A, Pintzas A. Epithelial-mesenchymal transition in cancer metastasis: mechanisms, markers and strategies to overcome drug resistance in the clinic. Biochim Biophys Acta. 2009; 1796: 75-90.

29. Schulte J, Weidig M, Balzer P, Richter P, Franz M, Junker K, Gajda M, Friedrich K, Wunderlich H, Östman A, Petersen I, Berndt A. Expression of the E-cadherin repressors Snail, Slug and Zebl in urothelial carcinoma of the urinary bladder: relation to stromal fibroblast activation and invasive behaviour of carcinoma cells. Histochem Cell Biol. 2012; 138: 847-860.

30. Li W, Mu D, Tian F, Hu Y, Jiang T, Han Y, Chen J, Han G, Li X. Exosomes derived from Rab27a-overexpressing tumor cells elicit efficient induction of antitumor immunity. Mol Med Rep. 2013; 8: 1876-1882. 